\title{
Clinical Study \\ Effect of Antihypertensive Therapy on SCORE-Estimated Total Cardiovascular Risk: Results from an Open-Label, Multinational Investigation-The POWER Survey
}

\author{
Guy De Backer, ${ }^{1}$ Robert J. Petrella, ${ }^{2}$ Assen R. Goudev, ${ }^{3}$ Ghazi Ahmad Radaideh, ${ }^{4}$ \\ Andrzej Rynkiewicz, ${ }^{5}$ and Atul Pathak ${ }^{6}$ \\ ${ }^{1}$ Department of Public Health, Ghent University Hospital, DePintelaan 185, 9000 Ghent, Belgium \\ ${ }^{2}$ Department of Family Medicine and Cardiology, Lawson Health Research Institute, 801 Commissioners Road East, \\ University of Western Ontario, ON, Canada, N6C 5J1 \\ ${ }^{3}$ Preventive Cardiology Clinic, Department of Internal Medicine, Medical University of Sofia, Sofia 1431, Bulgaria \\ ${ }^{4}$ Rashid Hospital, P.O. Box 4545, Oud Metha Road, Dubai, UAE \\ ${ }^{5}$ Department of Cardiology, Medical University of Gdañsk, M. Skłodowskiej-Curie 3a Street, 80-210 Gdañsk, Poland \\ ${ }^{6}$ Clinical Pharmacology Service, INSERM Unit 1048, Faculty of Medicine, University Hospital Toulouse, 31073 Toulouse, France
}

Correspondence should be addressed to Guy De Backer; guy.debacker@ugent.be

Received 19 March 2013; Revised 21 May 2013; Accepted 4 June 2013

Academic Editor: Kazuomi Kario

Copyright ( $\odot 2013$ Guy De Backer et al. This is an open access article distributed under the Creative Commons Attribution License, which permits unrestricted use, distribution, and reproduction in any medium, provided the original work is properly cited.

Background. High blood pressure is a substantial risk factor for cardiovascular disease. Design \& Methods. The Physicians' Observational Work on patient Education according to their vascular Risk (POWER) survey was an open-label investigation of eprosartan-based therapy (EBT) for control of high blood pressure in primary care centers in 16 countries. A prespecified element of this research was appraisal of the impact of EBT on estimated 10-year risk of a fatal cardiovascular event as determined by the Systematic Coronary Risk Evaluation (SCORE) model. Results. SCORE estimates of CVD risk were obtained at baseline from 12,718 patients in 15 countries (6504 men) and from 9577 patients at 6 months. During EBT mean $( \pm S D)$ systolic/diastolic blood pressures declined from $160.2 \pm 13.7 / 94.1 \pm 9.1 \mathrm{mmHg}$ to $134.5 \pm 11.2 / 81.4 \pm 7.4 \mathrm{mmHg}$. This was accompanied by a $38 \%$ reduction in mean SCORE-estimated CVD risk and an improvement in SCORE risk classification of one category or more in 3506 patients (36.6\%). Conclusion. Experience in POWER affirms that (a) effective pharmacological control of blood pressure is feasible in the primary care setting and is accompanied by a reduction in total CVD risk and (b) the SCORE instrument is effective in this setting for the monitoring of total CVD risk.

\section{Introduction}

Elevated blood pressure is a powerful contributor to total cardiovascular disease (CVD) risk. Clinical trials' experience supports the expectation of reduced cardiovascular risk from blood pressure control [1]. However, blood pressure control should not be applied in isolation. Rather, total CVD risk should be estimated in order to adapt the nature and intensity of preventive strategies to the circumstances of individual patients. A range of risk prediction models have been proposed for this purpose. One such is the
Systematic Coronary Risk Evaluation (SCORE) model [2]. This instrument, designed for use in primary prevention, estimates the total risk of fatal CVD events over the next 10 years as a function of age, gender, systolic blood pressure (SBP), total cholesterol, and smoking habits.

Eprosartan (Abbott Products Operations AG, Allschwil, Switzerland) is an orally administered, nonpeptide, angiotensin-receptor blocker widely approved within the European Union and in the USA for the treatment of hypertension. The Physicians' Observational Work on patient Education according to their vascular Risk (POWER) study created 
opportunities to evaluate (a) the effect of treatment with eprosartan on SBP in a very large cohort derived from countries with varying degrees of total CVD risk (and different healthcare systems) and (b) the effect of eprosartan-based therapy (EBT) on total CVD risk as quantified by the SCORE model.

Patients were recruited from 16 countries: Bahrain, Belgium, Bulgaria, Canada, Croatia, Greece, Republic of Korea, Kuwait, Poland, Qatar, Russia, Saudi Arabia, South Africa, Sweden, Thailand, and UAE. Participants from 15 of these countries were evaluated for changes in their SCORE risk status during the treatment phase of POWER. Framingham risk equations were used to monitor changes in CVD risk status among patients in Canada.

We report here the principal findings of changes in SCORE risk distribution during this large international survey. Data from the Canadian contingent are the subject of a separate report.

\section{Methods}

The design and methodology of POWER have been described in detail in a separate publication [3]. In brief, POWER was an open-label, postmarketing surveillance study of 6 months' duration. Participating physicians (general practitioners or cardiologists) collected data for at least five sequentially recruited patients with (a) newly diagnosed mild-tomoderate hypertension (mean sitting SBP >140 mmHg) who physicians proposed to treat with eprosartan or (b) existing hypertension considered insufficiently well controlled by current therapy and in whom eprosartan could be safely added.

The study protocol specified an initial regimen of eprosartan $600 \mathrm{mg} /$ day. This could be supplemented with another antihypertensive agent (preferably hydrochlorothiazide (HCTZ) $12.5 \mathrm{mg} /$ day) if the blood pressure response after 1 month of eprosartan monotherapy was considered insufficient.

Patients were recruited from the 15 SCORE-eligible countries and stratified according to their country's standing in the SCORE risk distribution. These assignments were made after discussions with national SCORE coordinators. Canada was excluded from these arrangements because in that country the Framingham risk equation was used to monitor changes in CVD risk status.

2.1. Ethical Considerations. The protocol of the POWER study was developed in conformity with existent rules and guidance for good clinical practice and the ethical conduct of research in humans, including the precepts of informed consent, and was subject to Institutional Review Board and/or Ethics Committee review and approval as required by local regulations and practice.

All patients were advised that they were free to withdraw from the study at any time and for any reason without prejudice to their subsequent medical care.

Although initiated in advance of the publication of STROBE recommendations for the conduct of observational research, POWER was fully consistent with the provisions of that guidance.

2.2. Statistics. The primary objective of POWER was to assess the absolute change in SBP in a large hypertensive population treated with EBT for 6 months.

Secondary efficacy variables included the absolute change in 10-year risk of fatal CVD assessed by SCORE from baseline to final visit. A chart-based SCORE estimate was generated centrally using the appropriate SCORE risk chart and individual patient data collected by physicians and recorded on case record forms.

Nominal qualitative variables were compared using the $\mathrm{Chi}^{2}$ test, ordinal qualitative variables were compared using the Wilcoxon test, and quantitative variables were compared using analysis of variance. Descriptive statistics were prepared for safety data on all patients who received at least one dose of study treatment.

\section{Results}

Between May 2005 and October 2009 a total of 28,369 patients were recruited in 15 countries. The derivation of a safety population of 28,055 patients and an intent-to-treat (ITT) cohort of 25,078 patients is shown in Figure 1.

3.1. SCORE Population Data. After exclusion of patients in whom the SCORE risk estimation was not applicable (patients with established CVD or diabetes) and of patients recruited in Canada, there remained 12,718 patients who constituted a primary prevention population to which the SCORE methodology was applicable (Figure 1). This contingent had an almost 1:1 distribution of men $(n=6504)$ and women $(n=6214)$, although the women were on average older (61.2 \pm 12 years versus $56.8 \pm 12.1$ years; $P<0.0001)$; a substantially greater proportion of women than men were 70 or more years old $(27.1 \%(n=1686)$ versus $15.4 \%(n=1001)$; $P<0.0001$ for overall age distribution). Overall, $26 \%$ of the patients were smokers; their mean cholesterol level was $5.50 \pm 0.87 \mathrm{mmol} / \mathrm{L}$, and $68.9 \%$ had a cholesterol level at baseline $>5.1 \mathrm{mmol} / \mathrm{L}$.

3.2. Blood Pressure Data. Baseline SBP/diastolic blood pressure (DBP) in the SCORE-eligible contingent was $160.2 \pm$ $13.7 / 94.1 \pm 9.1 \mathrm{mmHg}$; the intersex difference at baseline was $<2 \mathrm{mmHg}$. Mean pulse pressure (PP) was $66.15 \pm$ $14.01 \mathrm{mmHg}$, with a $2 \mathrm{mmHg}$ higher average value in women than in men. PP also increased with age, due primarily to a trend towards a slight decrease in DBP with age. The distribution of hypertension categories in these patients (Table 1) was in conformity with the $\approx 3: 1$ predominance of systodiastolic hypertension over isolated systolic hypertension seen in the overall ITT population [4].

Some $41.8 \%$ of patients in the SCORE ITT population were initially assigned to monotherapy $(n=5315)$; a further $33.0 \%(n=4193)$ were assigned to dual therapy and $22.4 \%(n=2847)$ were assigned to multidrug therapy (i.e., $\geq 3$ drugs). Combination details were not recorded for 


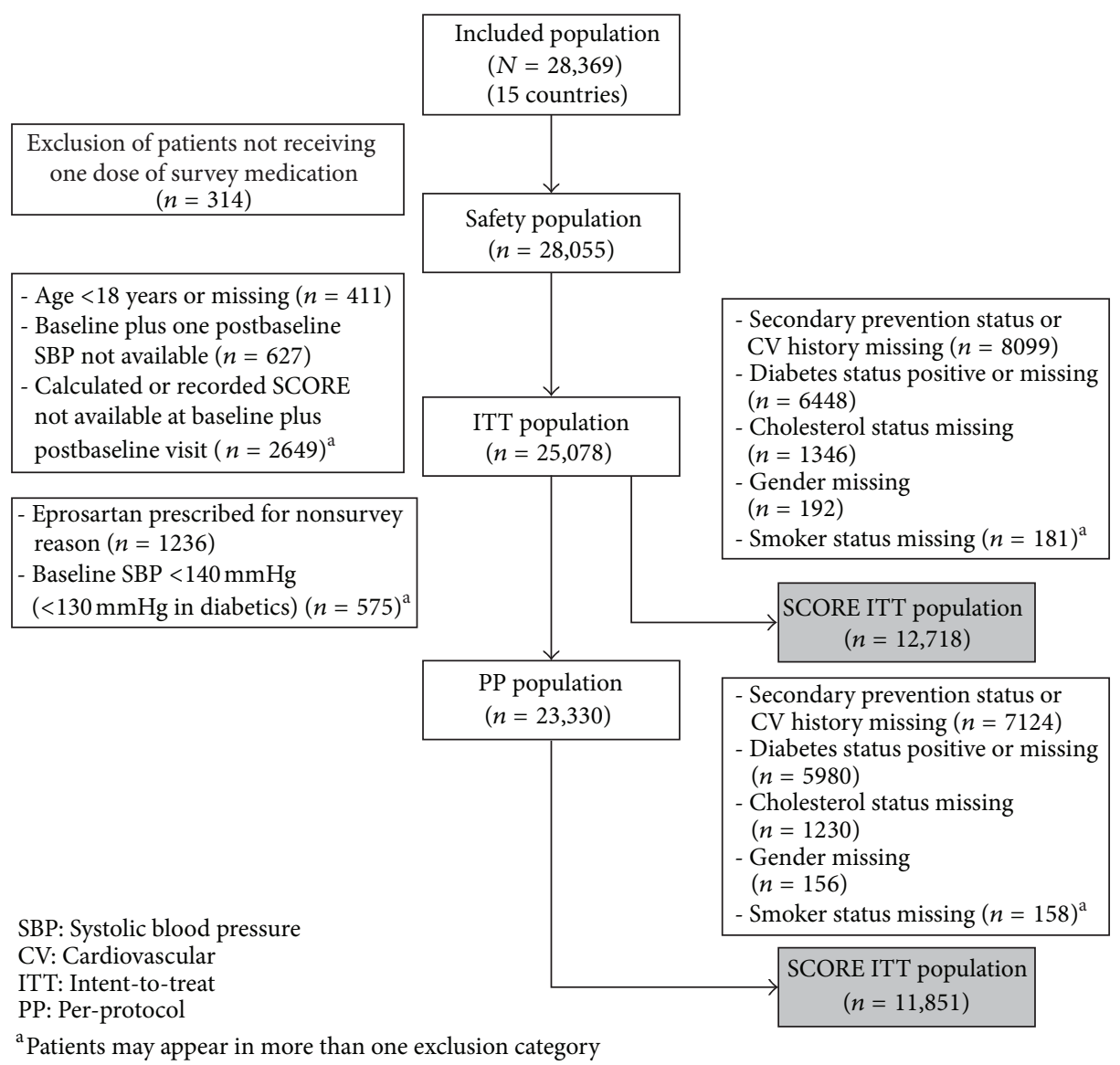

FIGURE 1: Patient disposition and derivation of the survey populations, including the SCORE ITT cohort.

TABLE 1: Hypertension classification in the SCORE-eligible contingent of POWER $(n=12,718)$.

\begin{tabular}{lccc}
\hline Patient population & $\begin{array}{c}\text { Male } \\
(n=6504)\end{array}$ & $\begin{array}{c}\text { Female } \\
(n=6214)\end{array}$ & $\begin{array}{c}\text { Total population } \\
(n=12,718)\end{array}$ \\
\hline Patients contributing data & 6477 & 6172 & 12,649 \\
Isolated systolic hypertension; $n(\%)$ & $1296(20.0)$ & $1515(24.5)$ & $2811(22.2)$ \\
Isolated diastolic hypertension; $n(\%)$ & $92(1.4)$ & $58(0.9)$ & $150(1.2)$ \\
Systodiastolic hypertension; $n(\%)$ & $5026(77.6)$ & $4529(73.4)$ & $9555(75.5)$ \\
No hypertension $(\mathrm{SBP}<140 \mathrm{mmHg}$ and DBP $<90 \mathrm{mmHg}) ; n(\%)$ & $63(1.0)$ & $70(1.1)$ & $133(1.1)$ \\
Missing values & 27 & 42 & 69 \\
\hline
\end{tabular}

SBP: systolic blood pressure; DBP: diastolic blood pressure.

363 patients. The most often recorded drugs supplementing eprosartan at baseline were diuretics (including a fixed-dose combination of eprosartan with HCTZ; 27.4\%; $n=3480$ ), beta-blockers (26.5\%; $n=3373)$, and calcium channel blockers (19.8\%; $n=2519)$. Proportionately, more women than men were prescribed a combination of eprosartan plus a non-HCTZ diuretic (19.3\% versus $12.6 \%$ ). The percentage of patients in receipt of combination therapy increased with age $\left(P<0.0001\right.$ by $\mathrm{Chi}^{2}$ test $)$.

The median duration of treatment was 182 days. During that time SBP and DBP declined by a mean of $-25.6 \pm 14.2 \mathrm{mmHg}$ and $-12.7 \pm 9.2 \mathrm{mmHg}$, respectively, to a mean value of $134.5 / 81.4 \mathrm{mmHg}$. Mean PP fell by $\approx 12.9 \mathrm{mmHg}$. A blood pressure response to therapy, defined as either SBP $<140 \mathrm{mmHg}$ and/or a reduction of SBP of $\geq 15 \mathrm{mmHg}$ or $\mathrm{DBP}<90 \mathrm{mmHg}$ and/or reduction of DBP of $\geq 10 \mathrm{mmHg}$, was recorded in $92.9 \%$ of patients; $62.9 \%$ of patients were classified as having normalized blood pressure at the end of observation, defined as SBP $<140 \mathrm{mmHg}$ and DBP $<90 \mathrm{mmHg}$.

Significant absolute changes in other risk factors were also observed after 6 months, including a reduction in body mass index of $-0.4 \pm 1.2 \mathrm{~kg} / \mathrm{m}^{2}(P<0.0001)$ and a $6.5 \%$ reduction in total cholesterol from $5.50 \pm 0.87 \mathrm{mmol} / \mathrm{L}$ to $5.09 \pm 0.74$ 
TABLE 2: Trends in total cholesterol distribution and smoking status during the POWER study.

\begin{tabular}{lcc}
\hline & $\begin{array}{c}\text { At baseline } \\
(n=12,718)\end{array}$ & $\begin{array}{c}\text { At final visit } \\
(n=9909)\end{array}$ \\
\hline & \multicolumn{1}{c}{ Cholesterol distribution; $n(\%)$} \\
$\leq 4.5 \mathrm{mmol} / \mathrm{L}$ & $1602(12.6)$ & $2067(20.9)$ \\
$4.5-5.1 \mathrm{mmol} / \mathrm{L}$ & $2351(18.5)$ & $2777(28)$ \\
$>5.1 \mathrm{mmol} / \mathrm{L}$ & $8765(68.9)$ & $5065(51.1)$ \\
\hline \multicolumn{3}{c}{ Smokers $(\%)$} \\
Smoking status: yes & 26 & 23.3 \\
\hline
\end{tabular}

$\mathrm{mmol} / \mathrm{L}(P<0.0001)$. Trends in the cholesterol distribution and smoker status during the study are shown in Table 2.

3.3. SCORE Data. The mean chart-based SCORE value was $6.0 \pm 5.8 \%$ at baseline, with some sex difference (mean SCORE: men $7.6 \pm 6.8 \%$, women $4.4 \pm 3.9 \%)$. The corresponding mean value on completion of observation was $3.5 \pm 3.5 \%$ (men $4.4 \pm 4.1 \%$, women $2.5 \pm 2.4 \%$ ). The overall mean absolute reduction was $-2.4 \pm 3.1 \%$ (men $-3.1 \%$, women $-1.7 \%$ ); the overall mean relative reduction was $-38.4 \%$ (men $-38.4 \%$, women $-38.3 \%)$. Absolute risk increased with age at baseline $(4.1 \pm 3.4 \%$ at age $50-59$ years, $10.9 \pm 6.4 \%$ at $\geq 70$ years) but the relative reduction was $\approx 36 \%$ across all age groups.

SCORE risk was stratified into four categories: low risk $<1 \%$; moderate risk 1-4\%; high risk 5-9\%; and very high risk $\geq 10 \%$. The SCORE risk distribution among these patients at baseline and at the end of observation is depicted in Figure 2. At both recording points, there were marked sex differences within this overall finding: at baseline $10.6 \%$ of women were classified as low risk compared with $2.2 \%$ of men, whereas $29.9 \%$ of men were classified as very high risk compared with $10 \%$ of women; at the final visit (based on $n=9577$ ), $17.1 \%$ of women were classified as low risk compared with $6.9 \%$ of men, whereas $8.1 \%$ of men were classified as being at very high risk compared with $1.9 \%$ of women (Figure 2 ).

Figure 3 illustrates the shifts in risk distribution for the 9577 patients who provided chart-based SCORE estimates at baseline and at the conclusion of observation.

Of the 1874 patients initially classified as being at very high risk, 75\% were reclassified to lower-risk categories at the completion of observation (Figure 3).

Among patients initially classified as high risk, $59.1 \%$ were reclassified as either moderate or low risk, while $20(\approx 0.7 \%)$ were reclassified as very high risk (Figure 3).

Most patients (87.9\%) initially estimated to be at moderate risk remained in that category at the end of observation. A further $11.6 \%$ of patients were reclassified as low risk and 25 patients $(<1 \%)$ were reclassified to higher-risk categories (Figure 3).

Almost all patients (99\%) initially classified as low risk were similarly classified at the completion of observation (Figure 3).

Overall, therefore, antihypertensive treatment for 6 months was associated with an improvement in SCORE risk of one category or more in $36.6 \%$ of patients $(n=$
TABLE 3: Summary of suspected adverse drug reactions (SADRs) and in-study deaths recorded during POWER.

\begin{tabular}{lc}
\hline Adverse event & $\begin{array}{c}\text { Number of events } \\
\text { (No. of patients) }\end{array}$ \\
\hline SADRs & $374(298)$ \\
SADRs leading to study discontinuation & $255(205)$ \\
Serious SADRs & $14(11)$ \\
Severe SADRs & $36(29)$ \\
Deaths & $5(5)$ \\
\hline
\end{tabular}

$3506)$, whereas 51 patients $(\approx 0.5 \%)$ were considered to have experienced a deterioration of one category or more.

In analysis stratifying patients by age, the proportion of patients classified as low or moderate risk increased from $51.3 \%$ at baseline to $70.7 \%$ at the final clinical visit (data not shown in detail). At baseline, $8.4 \%$ of patients aged 70 years or more $(n=227)$ were classified as moderate risk, $43.7 \%$ $(n=1175)$ were classified as high risk, and $47.8 \%(n=1285)$ as very high risk. At the completion of the observation phase, these proportions had shifted to $28.3 \%(n=529), 57.9 \%$ $(n=1082)$, and $13.9 \%(n=259)$, respectively.

An exploratory analysis comparing the CVD risk profile of the 9577 patients for whom SCORE data were available at baseline and at 6 months with the 3141 patients who had SCORE data available only at baseline identified no clinically relevant differences that might have influenced the SCORE findings (data not shown).

Among patients whose overall SCORE risk improved by at least one category, 97\% had a reduction in SBP of at least one grade, $37 \%$ achieved an improvement of at least one grade in cholesterol status, and 6\% reported stopping smoking during the study.

3.4. Safety Findings. Safety data were accrued from a population of 28,055 patients recruited in the countries that used SCORE methodology to calculate CVD risk (Figure 1). Within that population, a total of 374 events (in 298 patients) were classified as suspected adverse drug reactions (SADRs). Summary details of these SADRs, and of in-study deaths, appear in Table 3.

Two of the five deaths recorded were classified as SADRs. Causes of death in these cases comprised coma and cerebral bleeding. The deaths recorded as not being an SADR were attributed to lung embolism and ovarian cancer, unexpected death, and cerebrovascular accident in one patient each. The deaths associated with coma or cerebral bleeding were formally classified as suspect because a possible causal relation to use of study medication was not indicated by the investigators.

\section{Discussion}

In this open-label observational intervention of 6 months' duration, antihypertensive therapy based on eprosartan (with additional agents as considered necessary) was associated with a mean $-25.6 \pm 14.2 \mathrm{mmHg}$ reduction in SBP in a population with predominantly systodiastolic hypertension. 

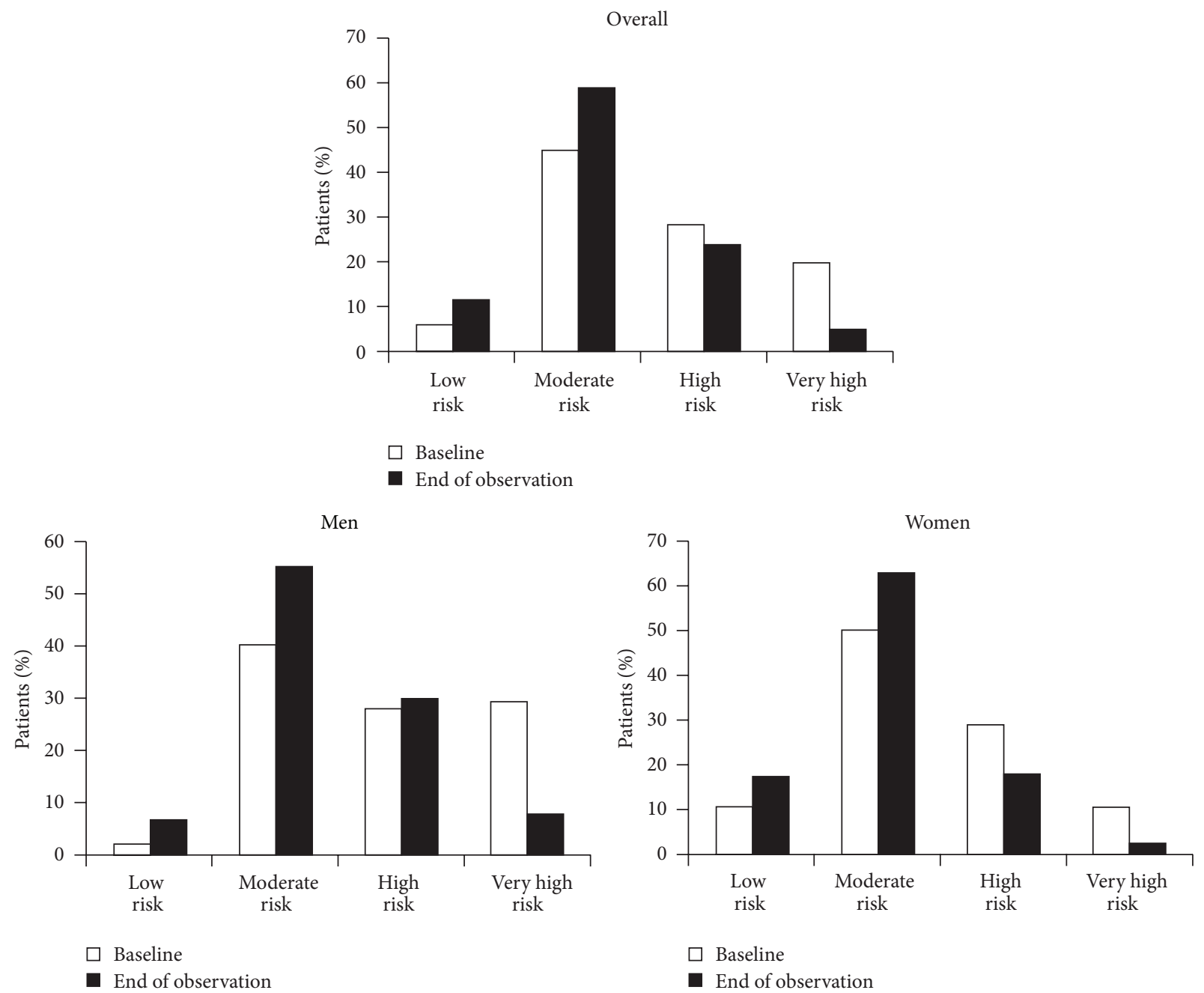

FIGURE 2: Chart-based estimates of SCORE risk distributions at baseline and at the end of observation, overall and by sex. Estimates based on $n=12,718$ at baseline and $n=9577$ at 6 months. Low risk $<1 \%$; moderate risk 1-4\%; high risk 5-9\%; very high risk $\geq 10 \%$.

Mean DBP and PP both fell by more than $10 \mathrm{mmHg}$. These reductions, especially in SBP, may be larger than what might have been reported in a controlled study but are in keeping with the high degree of innate variability in blood pressure. As an open-label, observational exercise, POWER does not offer the rigor of a controlled trial. However, it conforms to the provisions of the SCOPE principles for open-label research [5], and the wide geographical spread of participating countries provides some assurance against the operation of systematic biases with potential to distort the results.

Given the established relation between systemic arterial blood pressure and CVD risk, it might be expected that the blood pressure reductions observed in this study would be accompanied by improved overall CVD risk status, and this was indeed the case. Among $>9000$ patients with no baseline diagnosis of CVD or diabetes mellitus (i.e., a true primary prevention population), the period of EBT was characterized by improved SCORE-estimated risk status in $36.6 \%$ of the patients. Among 1874 patients initially classified as being at very high CVD risk (defined as 10 -year risk $\geq 10 \%$ ), 1406
(75\%) achieved improvements in SCORE status of at least one category by the end of the observation phase (Figure 3). Similarly, 1593 of 2694 patients (59.1\%) initially classified as being at high risk achieved improvements in SCORE status of at least one division by the end of the observation phase (Figure 3). Fewer than $1 \%$ of participants $(n=51)$ experienced a deterioration in SCORE risk status during the period of observation, a finding that may be seen as further evidence of a cause-effect relation between the reduction in blood pressure and the improvement in CVD risk status.

Reference to the SCORE risk charts (available at http:// www.escardio.org/communities/EACPR/toolbox/healthprofessionals/Pages/SCORE-Risk-Charts.aspx\#countries) reveals that for nonsmoking patients similar to ours (age $\approx 60$ years, SBP $\approx 160 \mathrm{mmHg}$, baseline total cholesterol $\approx 5 \mathrm{mmol} / \mathrm{L}$ ), relative risk varies several-fold and that an approximately $25 \mathrm{mmHg}$ reduction in SBP, as was observed in POWER, can be expected to bring the relative risk of those initially in the higher-risk categories much closer to that of their peers, who are at low relative risk. In 


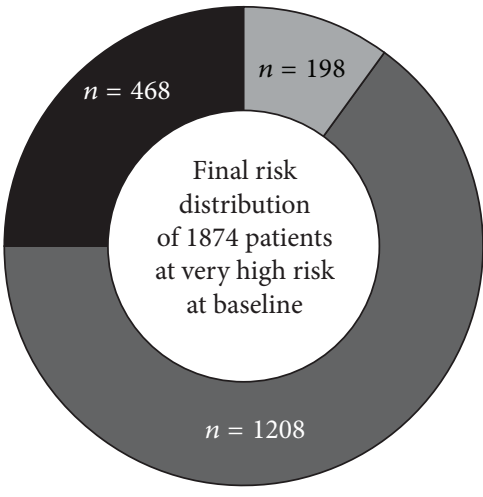

$n=0$

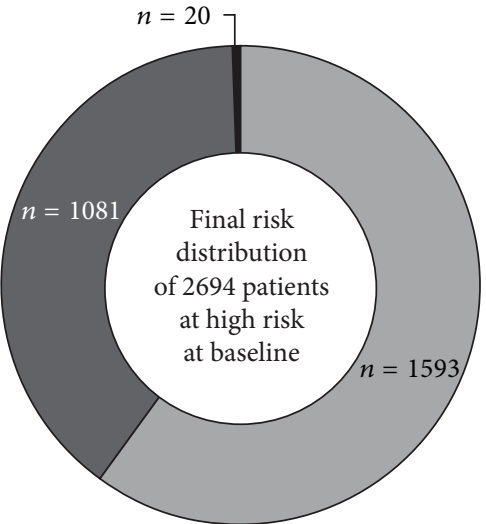

$n=0$

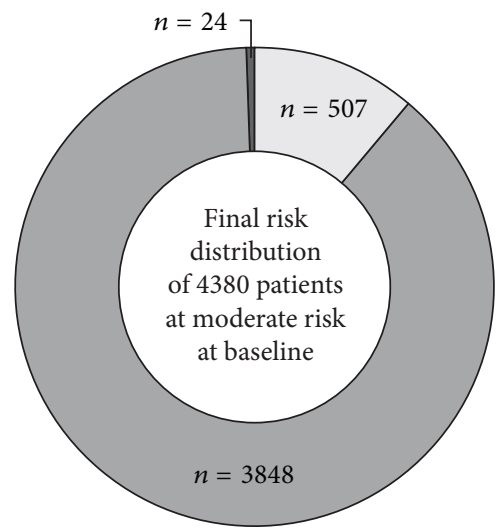

$n=1$

(a)

(b)

(c)

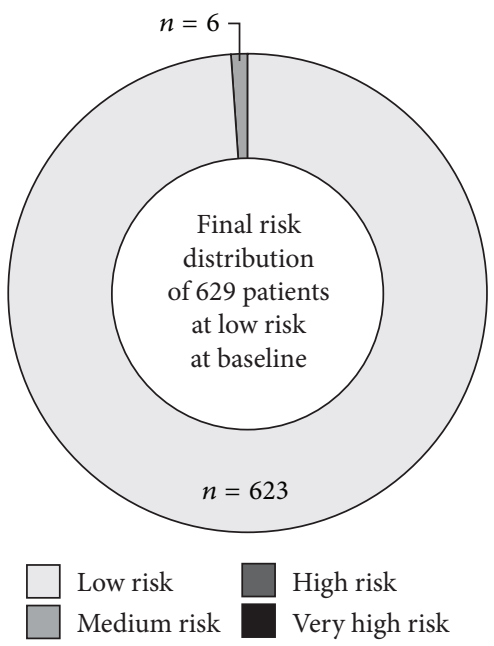

(d)

FIGURE 3: Shifts in SCORE risk distribution for the 9577 patients who generated chart-based estimates at baseline and at the conclusion of observation.

women-although less so in men-absolute 10-year risk may be brought to very low levels, as was the case in our patients.

Relative risk status may be further modified by smoking cessation. Physicians participating in POWER were mandated to counsel patients about smoking cessation but no standard instruments or programs were deployed for that purpose. Our data indicate that some $5 \%$ of patients reported smoking cessation during POWER. It may reasonably be assumed that few, if any, patients who stopped smoking did not report that fact. Hence, it seems likely that in this population smoking cessation played only a small part in the reductions in CVD risk status recorded via SCORE. As smoking cessation may be expected to halve the relative risk in patients such as ours and moderate the 10-year risk of a CVD event, the case for the addition of a smoking cessation program to control blood pressure as part of routine practice seems compelling. The work of Rodondi et al. offers one interesting perspective on this aspect of CVD risk management [6].
Inadequate control of hypertension is a major contributor to excess CVD mortality [7]. Our data reaffirm that effective pharmacological control of blood pressure is feasible in the primary care setting. It is thus a matter for concern that reports of widespread inadequate hypertension management persist in the medical literature [8-10]. We concur with Zannad et al. [11] that risk scoring systems such as SCORE have limitations but also agree that they provide a solid and accessible starting point for preventive cardiology involving minimum cost and complexity, and with no meaningful barriers to use. Given their relative ease of use, the SCORE charts are a practical resource for general practice. They are also to be preferred to unstructured physician estimates of risk [12].

Various proposals have been advanced for refining the accuracy and dependability of the SCORE technique [13-15]. However, application of the original SCORE charts appears to produce satisfactorily accurate risk estimates [16], and it seems to us that a much more significant impact on population CVD risk will be obtained from promoting a 
consistent and systematic use of the basic SCORE instrument (or similar) than from further attempts to refine the instrument without parallel efforts to encourage its use. Even then, the full value of using SCORE is likely to be realized only when it is integrated into a structured program of preventive activities. Experience from initiatives such as the Education and Coronary Risk Evaluation (EDUCORE) [17] and INterventions for COntrol of hyperTEnsion in CAtalonia (INCOTECA) [18] may prove instructive in shaping the delivery of primary preventive care. The need for continuing physician education also needs to be acknowledged [19].

\section{Conclusions}

In this open-label observational study, the use of EBT was associated with reduction in systemic blood pressure and associated CVD risk. The SCORE instrument was confirmed as an effective method for estimating and monitoring CVD risk in primary care.

\section{Disclosure}

AG reports funding by Abbott Products Operations AG, receipt of an honorarium from Abbott Products Operations AG and reimbursement for attending Abbott Products Operations AG symposia.

AP reports funding by Abbott Products Operations AG, receipt of an honorarium from Abbott Products Operations AG and reimbursement for attending Abbott Products Operations AG symposia.

AR reports speaker fees from Abbott, Astra-Zeneca, Servier and Merck Sharp \& Dohme.

GAR reports lecture fees from Abbott, Merck Sharp \& Dohme and NOVARTIS

GDeB reports research grants from Merck Sharp \& Dohme and speaker fees from Astra-Zeneca.

$\mathrm{RP}$ reports research grants from Pfizer, Astra-Zeneca, Novartis, Abbott and Sanofi-Aventis, and honoraria from Abbott, Novartis and Sanofi-Aventis for speaking engagements and symposia participation.

\section{Sponsor's Role}

The POWER study is supported financially by Abbott Products Operations AG, Hegenheimermattweg 127, 4123 Allschwil, Switzerland.

\section{Conflict of Interests}

None of the contributing authors has any affiliation to the SCORE methodology that confers any financial or other material gain.

\section{Authors' Contribution}

All the named authors contributed to the formulation of the study and the development of the study protocol. AG and AP were involved in the conduct of the study in their respective countries. All the named authors contributed to the analysis of the data and the development of the manuscript.

\section{Acknowledgments}

The authors wish to thank the physicians and patients who participated in the POWER survey. Preparation of this report was assisted by Hughes associates, 3 Collins St, Oxford, OX4 1XS, UK.

\section{References}

[1] F. Turnbull, "Effects of different regimens to lower blood pressure on major cardiovascular events in older and younger people: meta-analysis of randomised trials," British Medical Journal, vol. 336, no. 7653, pp. 1121-1123, 2008.

[2] R. M. Conroy, K. Pyörälä, A. P. Fitzgerald et al., "Estimation of ten-year risk of fatal cardiovascular disease in Europe: the SCORE project," European Heart Journal, vol. 24, no. 11, pp. 9871003, 2003.

[3] G. De Backer, R. J. Petrella, A. R. Goudev, G. A. Radaideh, A. Rynkiewicz, and A. Pathak, "Design and methodology of POWER, an open-label observation of the effect of primary care interventions on total cardiovascular risk in patients with hypertension," Fundamental and Clinical Pharmacology, vol. 27, pp. 210-215, 2013.

[4] A. Goudev, J.-P. Berrou, and A. Pathak, "Effect of eprosartanbased therapy on systolic blood pressure and total cardiovascular risk in a large international population: preliminary report of the POWER observational study," Vascular Health and Risk Management, vol. 8, pp. 563-568, 2012.

[5] E. Von Elm, D. G. Altman, M. Egger, S. J. Pocock, P. C. Gøtzsche, and J. P. Vandenbroucke, "The Strengthening the Reporting of Observational Studies in Epidemiology (STROBE) statement: guidelines for reporting observational studies," PLoS Medicine, vol. 4, no. 10, article e296, pp. 1623-1627, 2007.

[6] N. Rodondi, T.-H. Collet, D. Nanchen et al., "Impact of carotid plaque screening on smoking cessation and other cardiovascular risk factors: a randomized controlled trial," Archives of Internal Medicine, vol. 172, no. 4, pp. 344-352, 2012.

[7] E. Guallar, J. R. Banegas, E. Blasco-Colmenares et al., "Excess risk attributable to traditional cardiovascular risk factors in clinical practice settings across Europe-The EURIKA Study," BMC Public Health, vol. 11, article 704, 2011.

[8] Centers for Disease Control and Prevention (CDC), "Vital signs: awareness and treatment of uncontrolled hypertension among adults-United States, 2003-2010," Morbidity and Mortality Weekly Report, vol. 61, pp. 703-709, 2012.

[9] J. R. Banegas, E. López-García, J. Dallongeville et al., "Achievement of treatment goals for primary prevention of cardiovascular disease in clinical practice across Europe: the EURIKA study," European Heart Journal, vol. 32, no. 17, pp. 2143-2152, 2011.

[10] L. Missault, N. Witters, and J. Imschoot, "High cardiovascular risk and poor adherence to guidelines in 11069 patients of middle age and older in primary care centres," European Journal of Cardiovascular Prevention and Rehabilitation, vol. 17, no. 5, pp. 593-598, 2010.

[11] F. Zannad, G. De Backer, I. Graham et al., "Risk stratification in cardiovascular disease primary prevention-scoring systems, 
novel markers, and imaging techniques," Fundamental and Clinical Pharmacology, vol. 26, no. 2, pp. 163-174, 2012.

[12] É. Bruckert, G. Bonnelye, F. Thomas-Delecourt, L. André, and P.-H. Delaage, "Assessment of cardiovascular risk in primary care patients in France Évaluation du risque cardiovasculaire en médecine générale en France," Archives of Cardiovascular Diseases, vol. 104, no. 6-7, pp. 381-387, 2011.

[13] A. H. Merry, J. M. Boer, L. J. Schouten et al., "Risk prediction of incident coronary heart disease in the Netherlands: reestimation and improvement of the SCORE risk function," European Journal of Cardiovascular Prevention \& Rehabilitation, vol. 19, pp. 840-848, 2012.

[14] T. Sehestedt, J. Jeppesen, T. W. Hansen et al., "Risk prediction is improved by adding markers of subclinical organ damage to SCORE," European Heart Journal, vol. 31, no. 7, pp. 883-891, 2010.

[15] J. Wierzbicka-Chmiel, K. Mizia-Stec, M. Haberka, A. Chmiel, M. Mizia, and Z. Gasior, "The relationship between cardiovascular risk estimated by use of SCORE system and intima media thickness and flow mediated dilatation in a low risk population," Cardiology Journal, vol. 16, no. 5, pp. 407-412, 2009.

[16] D. De Bacquer and G. De Backer, "Predictive ability of the SCORE Belgium risk chart for cardiovascular mortality," International Journal of Cardiology, vol. 143, no. 3, pp. 385-390, 2010.

[17] I. Rodríguez-Salceda, E. Escortell-Mayor, M. Rico-Blázquez et al., "EDUCORE project: a clinical trial, randomised by clusters, to assess the effect of a visual learning method on blood pressure control in the primary healthcare setting," BMC Public Health, vol. 10, article 449, 2010.

[18] R. Vallès-Fernandez, M. Rosell-Murphy, O. Correcher-Aventin et al., "A quality improvement plan for hypertension control: the INCOTECA Project (Interventions for Control of hypertension in Catalonia)," BMC Public Health, vol. 9, article 89, 2009.

[19] R. B. Shaikh, E. Mathew, J. Sreedharan, J. Muttappallymyalil, S. A. Sharbatti, and S. A. Basha, "Knowledge regarding risk factors of hypertension among entry year students of a medical university," Journal of Family and Community Medicine, vol. 18, pp. 124-129, 2011. 


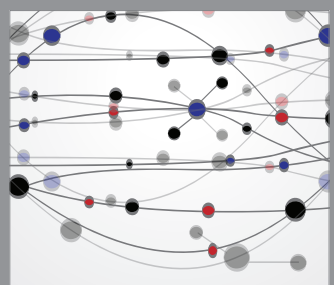

The Scientific World Journal
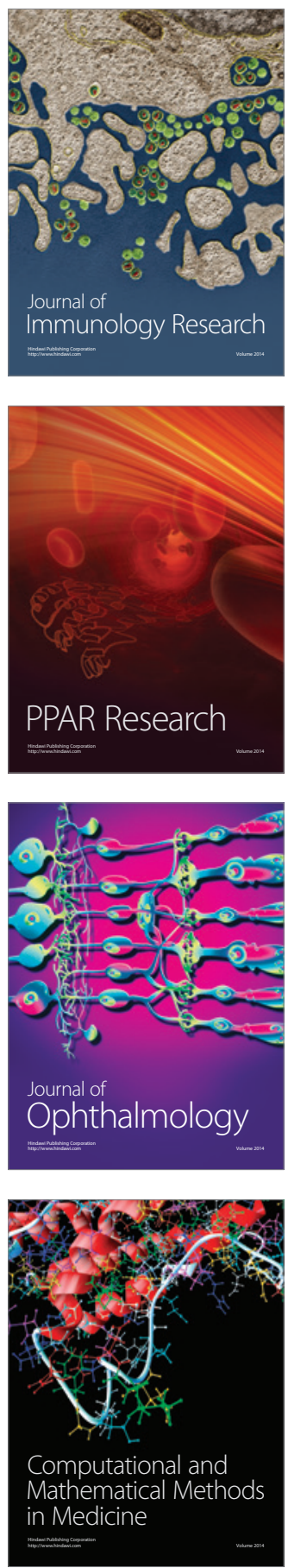

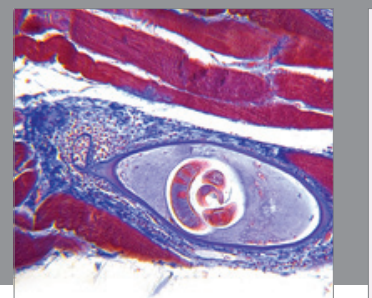

Gastroenterology

Research and Practice
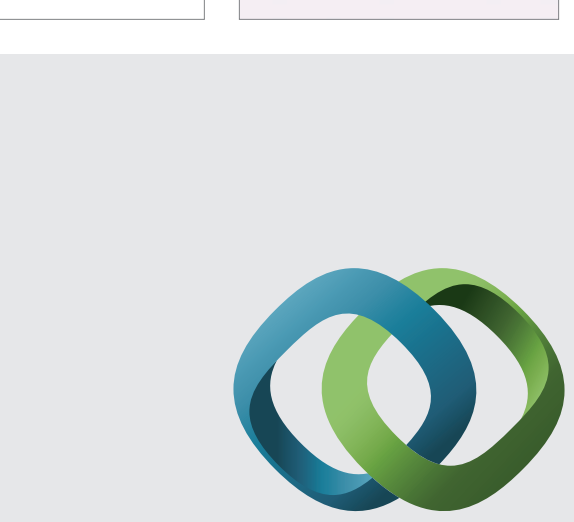

\section{Hindawi}

Submit your manuscripts at

http://www.hindawi.com
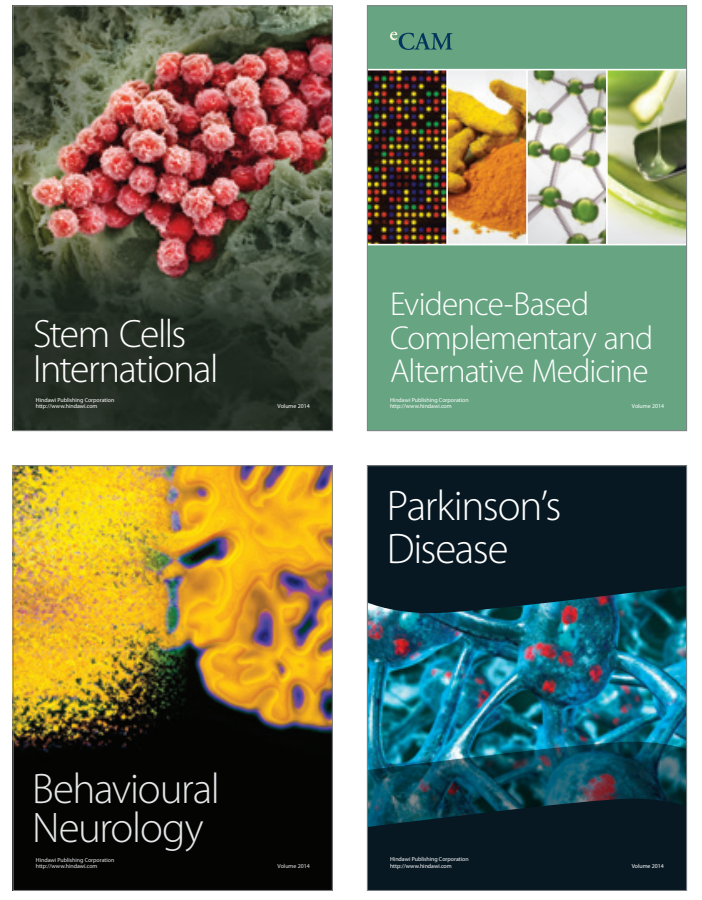
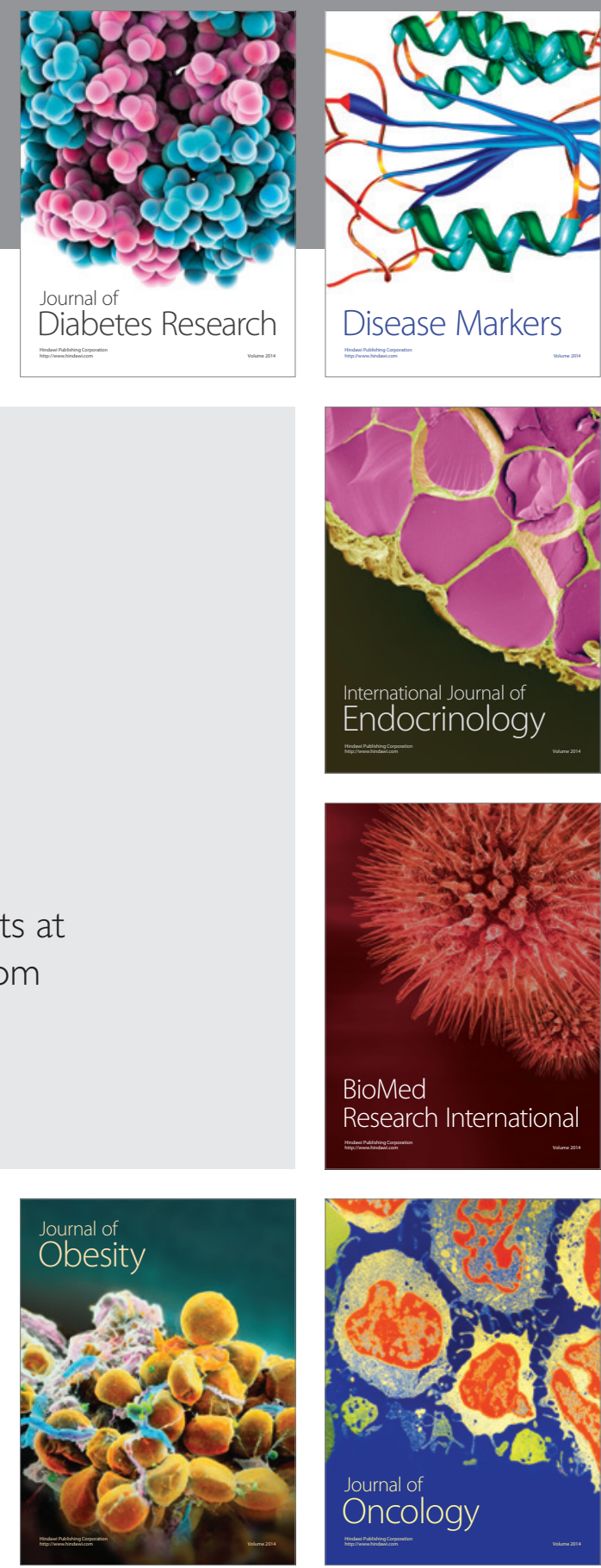

Disease Markers
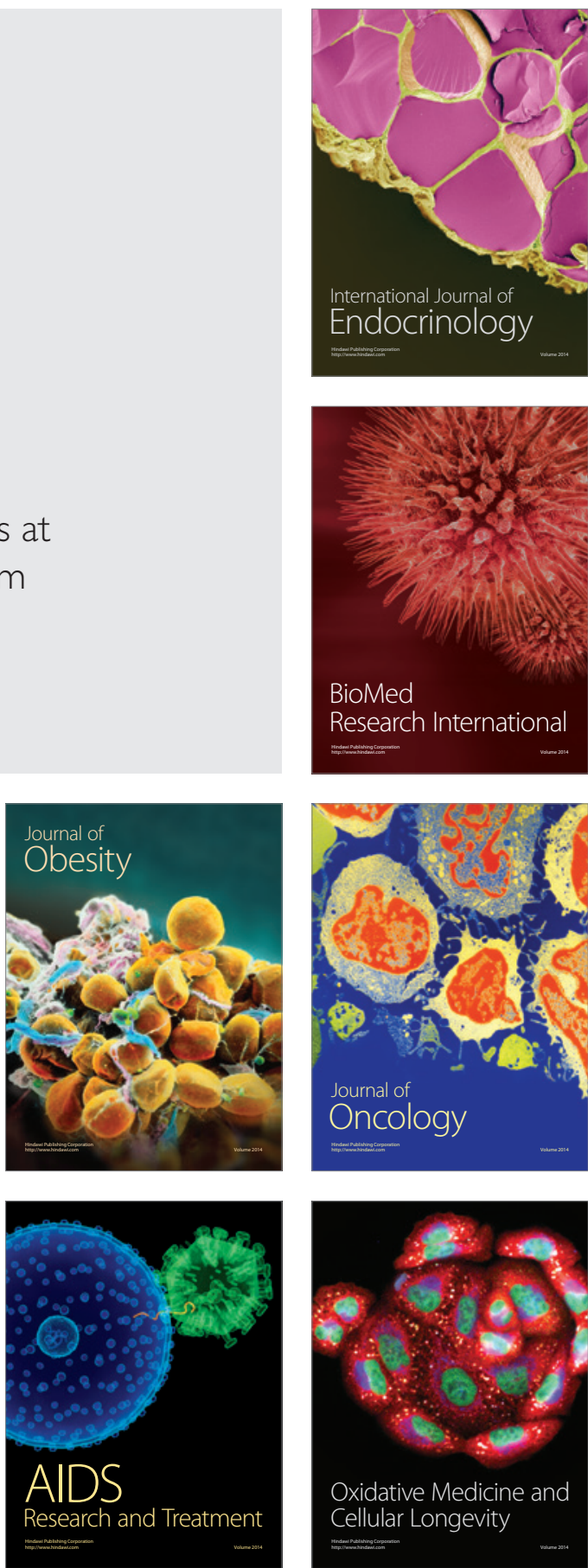